\title{
LA IMPORTANCIA CRECIENTE DE LAS FUERZAS DE OPERACIONES ESPECIALES EN ESTADOS UNIDOS Y SU INFLUENCIA EN EL RESTO DE PAÍSES DE LA OTAN
}

\author{
Raimundo Rodríguez ${ }^{1}$ y Javier Jordán ${ }^{2}$ \\ Universidad de Granada
}

\begin{abstract}
Resumen:
El artículo estudia la importancia creciente de las fuerzas de operaciones especiales en Estados Unidos. Este proceso se ha visto impulsado por factores relacionados con la naturaleza de la amenaza y con condicionantes de carácter social, político y económico. El proceso de innovación militar no sólo se ha traducido en el incremento de recursos humanos y materiales, sino en cambios profundos de carácter doctrinal y orgánico. El buen resultado de este tipo de unidades en las fuerzas armadas norteamericanas ha impulsado un proceso de difusión militar a otros países OTAN, que también están potenciando sus fuerzas de operaciones especiales.
\end{abstract}

Palabras clave: operaciones especiales, fuerzas armadas, Estados Unidos, innovación militar, OTAN, Afganistán.

Title in English: "The growing Importance of Special Forces in the US and its Influence in the Rest of the Countries"

\section{Abstract:}

The article examines the growing importance of special operations forces in the United States. This process has been driven by factors related to the nature of the threat and to social, political and economic constraints. The innovation process has not only resulted in increased human and material resources, but in profound changes in doctrinal and organizational character. The success of these units in the US military has driven a process of military diffusion to other NATO countries, which are also enhancing its special operations forces.

Keywords: Special operations forces, armed forces, United States of America, militay innovation, NATO, Afghanistan.

Copyright $@$ UNISCI, 2015.

Las opiniones expresadas en estos artículos son propias de sus autores, y no reflejan necesariamente la opinión de UNISCI. The views expressed in these articles are those of the authors, and do not necessarily reflect the views of UNISCI.

\footnotetext{
${ }^{1}$ Raimundo Rodríguez Roca es Teniente Coronel de Infantería (DEM), Estado Mayor del Ejército de Tierra. Sus líneas de investigación son: Innovación militar y fuerzas de operaciones especiales.

E-mail: rrodro7@et.mde.es.

${ }^{2}$ Javier Jordán es Profesor Titular de Ciencia Política en la Universidad de Granada, investigador asociado de la Universidad Autónoma de Chile. Sus líneas de investigación: Innovación militar, violencia política y terrorismo. E-mail: jjordan@ugr.es.
} 


\section{Introducción}

Es una constante a lo largo de la historia militar que los ejércitos traten de adaptarse, con más o menos éxito, a los cambios producidos en el entorno. La lucha contra redes terroristas de alcance global y contra actores insurgentes con vínculos transnacionales se ha convertido en uno de los principales cometidos de los ejércitos occidentales nada más comenzar el nuevo siglo. En Estados Unidos este proceso de cambio se ha concretado, entre otras medidas, en una atención cada vez mayor a las fuerzas de operaciones especiales. Y, de acuerdo con la lógica de los procesos de difusión militar, ese protagonismo también se ha extendido a otros países aliados, entre ellos España. Este artículo tiene por objeto analizar dicha dinámica.

\section{Las unidades de operaciones especiales en las fuerzas armadas norteamericanas: una tendencia al alza}

Las fuerzas armadas de los países occidentales están inmersas en diversos procesos de transformación, y parte de ellos lo constituye la adaptación y refuerzo de las capacidades de operaciones especiales. Este tipo de unidades se han convertido en un instrumento privilegiado en la lucha contra grupos terroristas e insurgentes. Muy en particular en la campaña liderada por Estados Unidos tras los atentados del 11 de septiembre de 2001 contra Al Qaeda y grupos afines, especialmente en escenarios de insurgencia como Irak y Afganistán.

En un informe presentado al Congreso de Estados Unidos después de la operación contra Osama Bin Laden en mayo de 2011 se destacaba la eficacia y evolución de las fuerzas de operaciones especiales tras una década de campañas:

"Las Unidades del Mando de Operaciones Especiales Conjunto, JSOC, y sus agrupaciones operativas se han convertido en el arma más letal y eficaz del gobierno de los Estados Unidos contra los terroristas y sus redes". ${ }^{3}$

"Las fuerzas de operaciones especiales y los operativos de inteligencia son las mejores fuerzas, las mejor adiestradas, las mejor equipadas y las mejor lideradas [...] Este éxito es consecuencia directa del liderazgo del presidente Obama y de las prioridades de seguridad nacional establecidas cuando llegó a la presidencia, así como a la luz verde que dio a las fuerzas de operaciones especiales este fin de semana".

Más allá del tono panegírico de ambos párrafos, lo cierto es que tanto las dos Administraciones Bush como las Administraciones Obama han confiado en las fuerzas de operaciones especiales para buscar y neutralizar a cuadros de alto nivel terroristas en los más diversos lugares del mundo. Esto ha sido posible, gracias a la transformación experimentada por el mando de operaciones especiales de Estados Unidos (US SOCOM) desde los atentados del 11 de septiembre de 2001, Muchos de esos cambios se han beneficiado de las tecnologías y conceptos doctrinales asociados a la Revolución en los Asuntos Militares, tan en boga en la década de $1990 .^{5}$ Hay dos ejemplos particularmente reseñables en este sentido:

\footnotetext{
${ }^{3}$ Congressional record, House of Representatives, Proceedings and debates of the $112^{\text {th }}$ Congress, first session vol. 157 Washington, May 2, 2011 no. 57. Library of Congress. p. H2928

${ }^{4}$ Congressional record. Senate, Proceedings and debates of the $112^{\text {th }}$ Congress, first session vol. 157 Washington, May 2, 2011 no. 57. p. S2559

${ }^{5}$ Lindsay, John R.: "Reinventing the Revolution: Technological Visions, Counterinsurgent Criticism, and the Rise of Special Operations", Journal of Strategic Studies, vol. 36, no 3 (2013), pp. 422-453.
} 
- Las operaciones terrestres en Afganistán durante los seis primeros meses de la campaña fueron dirigidas o realizadas por unidades especiales. Las fuerzas convencionales se emplearon en apoyo de éstas, y en determinadas misiones estuvieron bajo el mando de las fuerzas de operaciones especiales. Ello supuso un cambio innovador con respecto a la estructura tradicional donde las fuerzas de operaciones especiales actúan en apoyo de las unidades convencionales. La situación volvió a la 'normalidad' durante la operación Anaconda (marzo de 2002), cuando las unidades de operaciones especiales asumieron su rol habitual de multiplicador de fuerza del ejército convencional. Este cambio doctrinal y orgánico fue posible gracias a las tecnologías asociadas a la llamada revolución tecnológica militar. ${ }^{6}$

- Los cambios operados en el Joint Special Operations Command (JSOC), uno de los componentes del US SOCOM) en la neutralización de cuadros insurgentes y terroristas. Se trata de un tema justificadamente polémico por los abusos a prisioneros cometidos por el JSOC y por el modo de actuar de esta unidad en países con los que Estados Unidos no estaba en guerra. ${ }^{7}$ Pero analizado exclusivamente desde el punto de vista de nuestro estudio, lo que consiguió el General Stanley A. McChrystal (jefe del JSOC entre 2003 y 2008) encaja claramente en el concepto de innovación militar ${ }^{8}$. Una innovación además disruptiva, pues supuso una mejora sustancial en un modo de operar y combatir que hasta ese momento no había sido particularmente valorado. ${ }^{9}$

Exponer en detalle la innovación experimentada en el JSOC requeriría otro artículo. Baste ahora con señalar que el General Stanley A. McChrystal aplicó el principio de que para derrotar a una red (insurgente o terrorista) era necesaria otra red (la creada en torno al JSOC). Una idea que justificó en un artículo publicado en la revista Foreign Policy en 2011 a raíz de sus experiencias de Irak y Afganistán. ${ }^{10}$ Dicho principio ya había sido expresado unos cuantos años antes por John Arquilla y David Ronfeldt al teorizar sobre la netwar. ${ }^{11}$

Gracias a los avances tecnológicos en materia de inteligencia y transmisión de información y a una serie de cambios doctrinales y orgánicos adaptados a ellas, el JSOC fue capaz de aplicar el ciclo Find, Fix, Finish, Exploit, and Analyze de manera reiterada: los analistas encontraban a los cuadros de mando insurgentes mediante inteligencia humana y de señales, los operadores de drones fijaban el blanco, los equipos de operaciones especiales los detenían o mataban, y simultáneamente otros especialistas procesaban la información capturada en la operación (agendas de teléfonos, discos duros, detenidos, mapas, etc) y se la entregaban a los analistas que buscaban nuevos objetivos para repetir el ciclo. Un sistema que

\footnotetext{
${ }^{6}$ Lee, Gordon T. "Hard-Shelled, SOF-Centered. The Synergy of Might and Mind", RAND Review (Verano 2002).

${ }^{7}$ Scahill, Jeremy (2013): Dirty Wars: The World Is a Battlefield, New York, Nation Books.

${ }^{8}$ Rosen, Stephen Peter (1994): Winning the Next War: Innovation and the Modern Military, Ithaca (NY), Cornell Universty Press, p. 34; Posen, Barry, R. (1984): The Sources of Military Doctrine: France, Britain and Germany between the World Wars, Ithaca (NY), Cornell University, pp. 29-30.

${ }^{9}$ Pierce, Terry C. (2004): Warfighting and Disruptive Technologies. Disguising Innovation, New York, Frank Cass, p. 1 .

${ }^{10}$ McChrystal, Stanley, "It Takes a Network. The new front line of modern warfare", Foreign Policy, 21 de febrero de 2011.

${ }^{11}$ Arquilla, John y Ronfeldt, David: "Information, Power and Grand Strategy: In Athena's Camp-Section 1", en Arquilla, John y Ronfeldt, David (1997): In Athena's Camp. Preparing for Conflict in the Information Age, Santa Monica, RAND, pp. 141-171.
} 
se ha extendido a las fuerzas de operaciones especiales de otros países occidentales, e incluso a las fuerzas convencionales norteamericanas ${ }^{12}$.

Mediante esta innovación las fuerzas de operaciones especiales han simplificado su cadena de toma de decisiones, han adoptado una organización más plana y se han integrado y colaborado con diversas agencias de inteligencia. ${ }^{13}$ Todo ello, les ha permitido, en palabras del General McChrystal, acomodarse a la "velocidad de la guerra"14, en referencia a su adaptación a la velocidad de operar de los grupos terroristas e insurgentes quienes hacían gala de mayor flexibilidad y libertad de acción a la hora de aplicar y variar sus procedimientos de combate. McChrystal y sus colaboradores fueron los artífices de este proceso de cambio doctrinal y orgánico ${ }^{15}$.

Esas innovaciones han requerido una inversión presupuestaria desconocida hasta ahora para este tipo de fuerzas. En once años se incrementaron en un $450 \%$ los presupuestos dedicados a las fuerzas de operaciones especiales. En 2001, el Mando de Operaciones Especiales de Estados Unidos, USSOCOM recibía 2.3 mil millones de dólares y en 2012 casi lo había multiplicado por cinco alcanzado la cifra de 10,5 mil millones ${ }^{16}$. El incremento de recursos ha supuesto cierta elevación de la estatura del US SOCOM en la distribución de poder relativo dentro del Pentágono ${ }^{17}$. También ha contribuido a ello el aumento sustancial del número de generales y almirantes en puestos de alta responsabilidad, que pasó de apenas una docena a unos setenta en solo diez años ${ }^{18}$. Ello ha supuesto una mayor representatividad de la comunidad de operaciones especiales en las fuerzas armadas norteamericanas y, al igual que sucede en otros procesos de innovación militar, contribuye a institucionalizar los avances logrados en los últimos años.

\footnotetext{
${ }^{12}$ Robinson, Linda: "The Future of Special Operations. Beyond Kill and Capture", Foreign Affairs, vol. 91, nº 6 (2012), pp. 110-122.

${ }^{13}$ Robinson, Linda: "The future of Special Operations Forces", Council Special Report, n 66 (2013), Council of Foreign Relations, New York, pp 10-21, en http://i.cfr.org/content/publications/attachments/Special_Operations_CSR66.pdf.

${ }_{14}^{14}$ McChrystal, Standley (2013): My Share of the Task, New York, Penguin Group, p. 214.

15 Army Doctrine Reference Publication: Army Special Operations Forces Operational Methodology, ADPR-305, (31 de agosto 2012), pp. 3-10

${ }^{16}$ Feickert, Andrew: US Special Operations Forces (SOF): Background and issues for Congress. CRS Report for Congress, (28 de abril de 2015), p.8.

${ }^{17}$ Robinson, "The Future of Special Operations. Beyond Kill and Capture", op.cit.

${ }^{18}$ Robinson, "The Future of Special Operations Forces", op. cit., p 4.
} 
Grafico 1. Presupuestos asignados al US SOCOM entre los 2001 y 2013

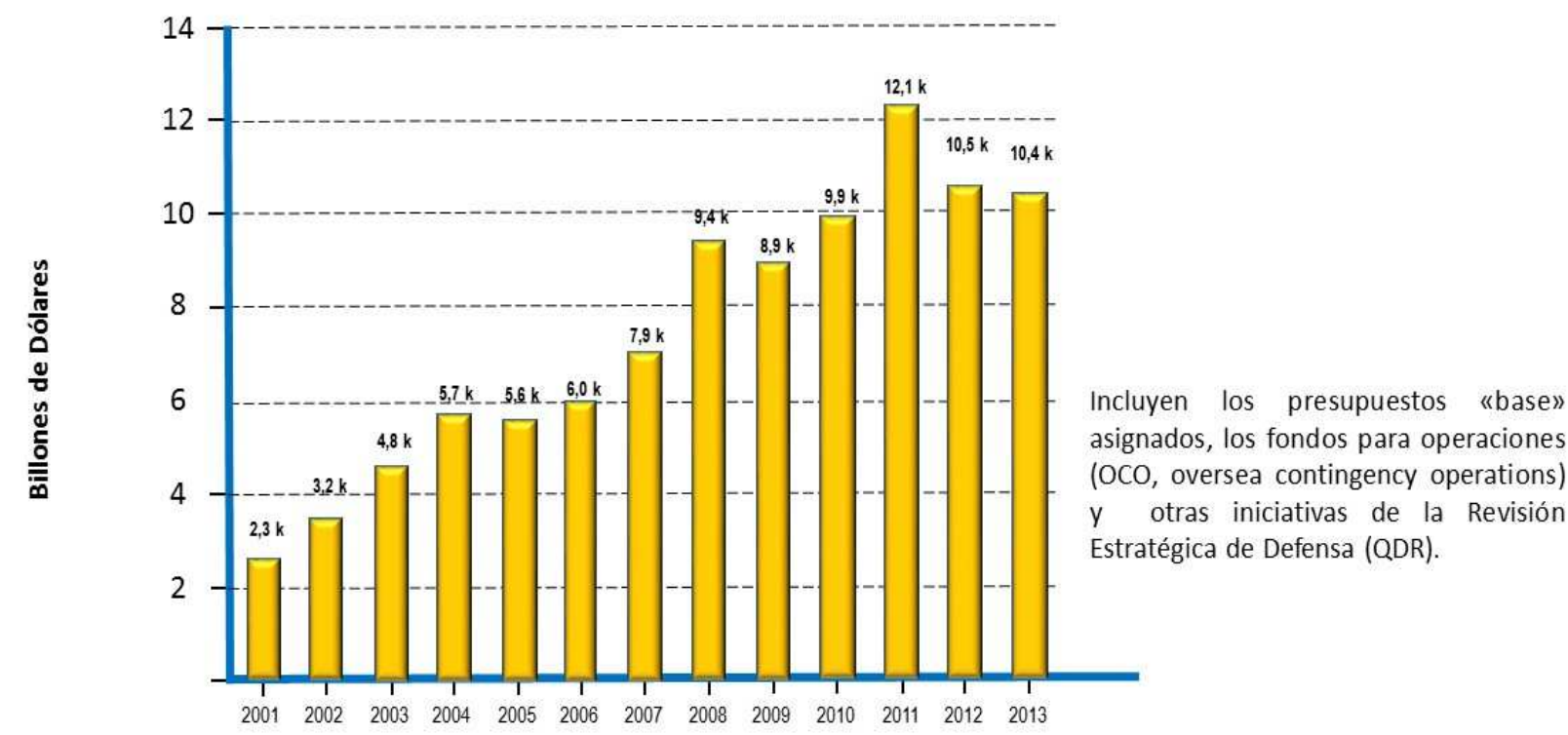

Años 2001-2013

Fuente: elaboración propia basada en datos de US SOCOM a través de su página oficial, más recopilación de los informes elaborados por Feickert, Andrew. US Special Operations Forces (SOF): Background and issues for Congress, CRS Report for Congress, publicados anualmente entre 2001 y 2013.

Pero en términos relativos, el coste real de las operaciones especiales ha sido muy escaso comparado con la magnitud total de los recursos invertidos en las operaciones Enduring Freedom e Iraqui Freedom. En 2013, uno de los años en los que el presupuesto para las fuerzas de operaciones especiales se encontraba en el punto más alto desde el inicio de la guerra en Afganistán, las fuerzas del US SOCOM recibieron el 1,7 \% del presupuesto total del Pentágono. Tanto por ciento que ascendía a $4 \%$ si se le añadían las aportaciones anuales del US Army y de la US Navy ${ }^{19}$. Se trata por tanto de unas fuerzas muy rentables desde el punto de vista de coste eficacia. Algo que les favorece en un escenario de restricciones presupuestarias, como el que viven a día de hoy las fuerzas armadas de Estados Unidos y de la mayoría de los países aliados.

En términos de efectivos el incremento presupuestario y la demanda operativa se han traducido en el crecimiento del US SOCOM en un $112 \%$. De unos 33.000 militares en 2001 hasta casi 70.000 a comienzos de $2015^{20}$. Las fuerzas de operaciones especiales de Estados Unidos desplegadas en todo el mundo han pasado de cerca de 5.000 en 2001, a unos 13.000 en 2011, un aumento de aproximadamente un $160 \%$. Parte importante de esa escalada se debió a las operaciones militares en Afganistán.

\footnotetext{
${ }^{19}$ Weisgerber, Marcus: "Peeling the Onion Back on the Pentagon's Special Operations Budget", Defense One News, 27 de enero de 2015, en http://www.defenseone.com/management/2015/01/peeling-onion-backpentagons-special-operations-budget/103905/.

${ }^{20}$ Turse, Nick: "Special Ops Goes Global", TomDispatch, 7 de enero de 2014, en http://www.tomdispatch.com/blog/175790/tomgram\%3A nick turse, special_ops_goes_Global; Turse, Nick: "The Golden Age of Black Ops: Special Ops Missions Already in 105 Countries in 2015", TomDispatch, 20 de enero de 2015, en http://www.truth-out.org/news/item/28633-the-golden-age-of-black-ops-special-ops-missionsalready-in-105-countries-in-2015.
} 
Grafico 2. Incremento de personal en el Mando de operaciones especiales de Estados Unidos (USSOCOM) desde el año 2001 al 2015

Incremento de personal en USSOCOM DESDE 2001 A 2015

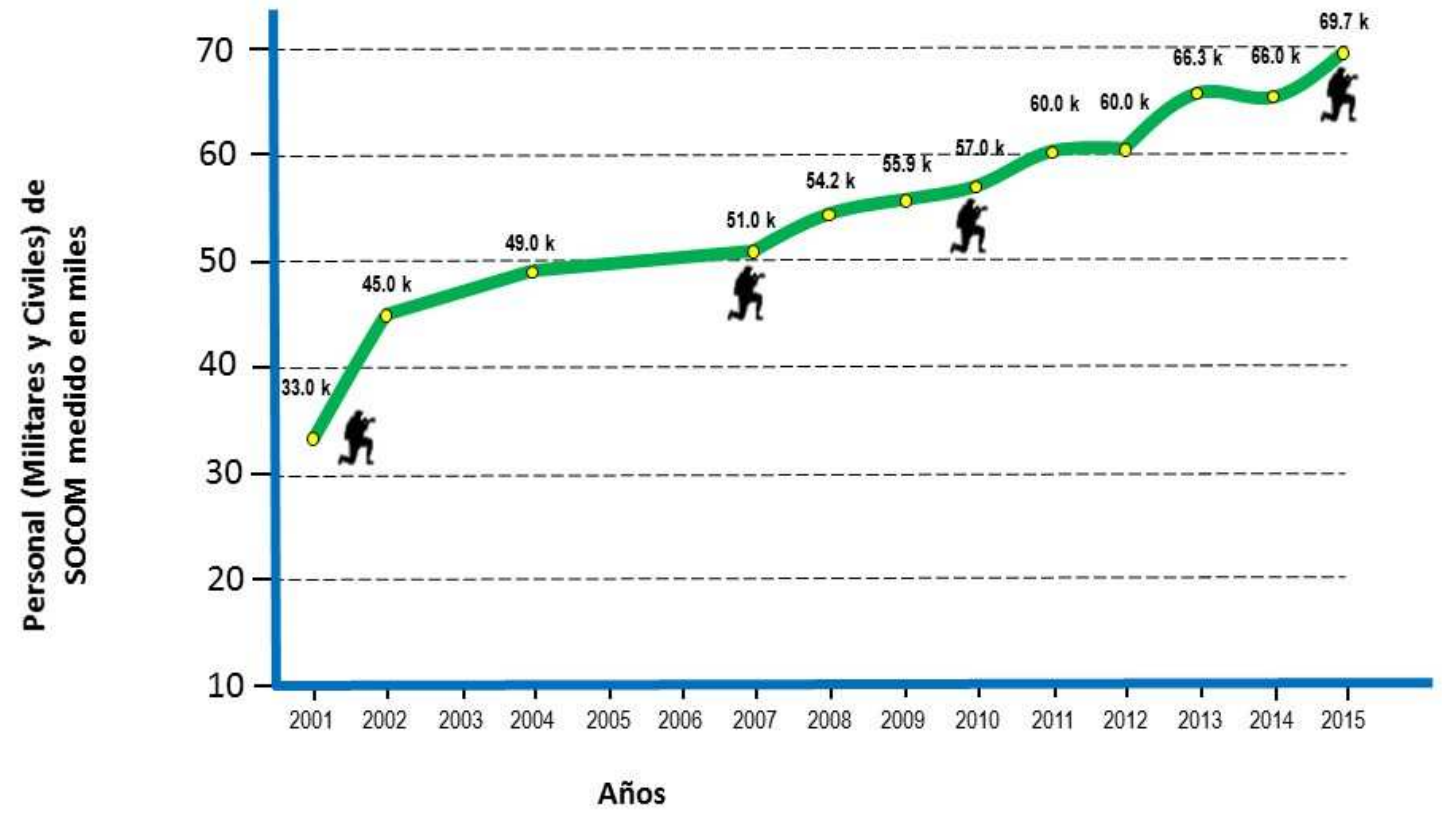

Fuente: elaboración propia basada en datos de US SOCOM a través de su página oficial, más recopilación de los informes elaborados por Feickert, Andrew, US Special Operations Forces (SOF): Background and issues for Congress, CRS Report for Congress, publicados anualmente entre 2001 y 2015.

Linda Robinsón, analista de la RAND Corporation, ofrece cantidades mayores que difieren ligeramente de las fuentes de US SOCOM, señalando que en su momento de apogeo, a partir de 2010, solo los "operadores" desplegados en Afganistán eran unos 13.700, con un apoyo directo de 17.000 , de los cuales unos 9.000 pertenecían a fuerzas de helicópteros. ${ }^{21}$

La disparidad se debe a que las cifras reales suelen variar de las oficiales. Estas últimas presentan cantidades acordes a los límites autorizados por el Congreso, mientras que en la práctica existe un margen debido a las fuerzas que entran y salen del teatro de operaciones. Los relevos de fuerzas se realizan de manera escalonada y hay momentos que ambas fuerzas coinciden durante los periodos de trasferencia de autoridad.

También hay otras explicaciones, como por ejemplo lo sucedido en Afganistán en septiembre de 2010. Durante el periodo de despliegue de capacidades, consecuencia del incremento de fuerzas que realizaba Estados Unidos para acelerar las condiciones necesarias para la salida de Afganistán, el Pentágono detectó un pequeño desajuste de fuerzas. Según el Departamento de Defensa, se había desplegado un $10 \%$ de fuerzas superior a lo autorizado por el Congreso. ${ }^{22}$ Se debía a que gran parte de los dividendos de fuerza originados por retirar medios y recursos de Irak se desplegaron directamente sin pasar por el territorio nacional norteamericano. El periodo de armonización se extendió por cerca de seis meses y afectó de manera desigual a cada área de responsabilidad de los Mandos Regionales de Afganistán. El ajuste de fuerzas tuvo impacto en los planes de aquel momento, ya que desde que el General

\footnotetext{
${ }^{21}$ Robinson, Linda (2013): One Hundred Victories: Special Operation and the Future of Warfare, New York, Public Affairs, capítulo 13.

${ }^{22}$ Belasco, Amy: "The Cost of Iraq, Afghanistan, and Other Global War on Terror Operations Since 9/11", CRS Report for Congress, 2 de septiembre de 2010.
} 
McChrystal se había hecho cargo del mando de ISAF en 2009, las revisiones de planes de campaña se habían diseñado de una forma más realista, teniendo en cuenta el aumento de medios en teatro y las nuevas posibilidades de hacer más que brindaban las nuevas capacidades $^{23}$.

Como consecuencia, el número de operadores desplegados permanentemente alcanzó unas cifras muy elevadas para tratarse de un personal altamente especializado y por tanto, un recurso limitado que se tardaba mucho tiempo en generar y en tener preparado para el combate. En 2012, el entonces jefe del US SOCOM, Almirante William McRaven, afirmaba que su mando incluía 66.000 efectivos de los cuales 44.000 eran potencialmente desplegables, y de ellos unos 33.000 pertenecían a unidades tácticas, con capacidad de ejecución de acciones en el terreno. De los operadores, en ese momento entre 10.000 y 12.000 , estaban desplegados simultáneamente en unos 79 países, 7.500 de ellos en Afganistán y el resto en otros lugares. Es decir, un tercio de sus 33.000 operadores estaban permanentemente desplegados fuera de Estados Unidos, si bien solo uno de esos escenarios, Afganistán, concentraba la mayoría de los recursos ${ }^{24}$.

De este modo, se ha producido un aumento continuado en el volumen de fuerzas de operaciones especiales sin precedentes en la historia militar norteamericana. Un aumento que ha ido vinculado a nuevas prioridades de gasto de defensa, recogidas en documentos periódicos de revisión de capacidades militares y en cambios legislativos para regular el empleo de estas fuerzas. ${ }^{25}$ En poco más de diez años se ha introducido un nuevo modelo estratégico de empleo de este tipo de fuerzas, con cambios en responsabilidades y cometidos, nuevas doctrinas, cambios en los métodos de adiestramiento, cambios en procedimientos, así como cambios y creación de nuevas estructuras orgánicas para potenciar el empleo de las fuerzas de operaciones especiales. Una innovación en el empleo de la fuerza a la que, como veremos a continuación, se han ido sumando la mayoría de los miembros OTAN.

\section{Consecuencias sobre las fuerzas de operaciones especiales de los aliados OTAN y otros países occidentales}

El éxito de un ejército a la hora de innovar suele poner en marcha procesos de emulación por parte de otras fuerzas armadas, impulsada por una de las tres siguientes razones: responder a los cambios del entorno estratégico tratando de estar a la altura de eventuales competidores, ser capaz de interoperar con ejércitos aliados, o fortalecer la identidad del ejército. ${ }^{26}$

La emulación puede estar justificada por un cálculo racional en la medida en que se trata de asumir un cambio que ya ha sido desarrollado y experimentado con éxito por las fuerzas armadas de otros país o países. Desde esa perspectiva la emulación es una estrategia eficiente, pues ahorra los costes que conlleva todo proceso de ensayo y error. Al mismo tiempo, también es racional si la innovación que se importa es necesaria y se cuenta con los

\footnotetext{
${ }^{23}$ Información del Teniente Coronel Jefe de la Sección de J5, Planes, en el Cuartel General del mando Regional Oeste de ISAF en Herat, Afganistán, Octubre 2010.

${ }^{24}$ Entrevista en el Aspen Security Forum al Almirante Willian McRaven Jefe del USSOCOM publicada en YouTube el 24 de septiembre de 2012, en http://www.youtube.com/watch?v=pR1Nkp5JdSc.

${ }^{25}$ Department of Defense: "Quadrennial Defense Review Report" (Febrero de 2006); Department of Defense: "Quadrennial Defense Review Report" (Febrero de 2010), Department of Defense: Quadrennial Defense Review Report (Marzo de 2014).

${ }^{26}$ Jordán, Javier: "¿Qué factores impulsan la innovación militar?", Análisis GESI, 12/2014 (30 de junio de 2014), en

http://www.seguridadinternacional.es/?q=es/content/\%C2\%BFqu\%C3\%A9-factores-impulsan-lainnovaci\%C3\%B3n-militar.
} 
recursos necesarios para asumirla. La generalización de las emulaciones da lugar a procesos de difusión militar. Según Michael C. Horowitz, la difusión militar se produce cuando un proceso de innovación alcanza un nivel de progreso que le permite 'debutar' o demostrar su efectividad tanto en la guerra como en tiempo de paz. Es en ese momento cuando el resto de actores cuentan con información suficiente para comprender el significado de esa innovación concreta y, en consecuencia, algunos de ellos se sienten impulsados a emularla. Otros, sin embargo, no lo hacen porque no lo necesitan, no cuentan con los recursos necesarios para ello o porque sus organizaciones militares no favorecen el cambio. ${ }^{27}$

En el caso que estamos analizando la importancia creciente que presta Estados Unidos a sus fuerzas de operaciones especiales ha tenido un eco efectivo en muchos de sus aliados OTAN, poniendo en marcha procesos de difusión en sus respectivas fuerzas armadas que podemos relacionar fundamentalmente con dos conjuntos de factores. Por un lado, la adaptación a un entorno estratégico caracterizado por conflictos asimétricos e híbridos como son a día de hoy por ejemplo los de Siria, Irak, Libia o Mali. Y, por otro, la necesidad de operar conjuntamente con las fuerzas de operaciones especiales norteamericanas en el marco de la OTAN, algo que se ha hecho extensivamente en Afganistán.

De este modo, en poco más de una década, ha tenido lugar un proceso de difusión que se ha traducido en la potenciación de este tipo de fuerzas y en la creación de nuevas estructuras orgánicas. En la mayoría de los casos han sido estructuras de carácter conjunto.

Cuadro 1. Mandos de Operaciones especiales creados de 1980 a 2001

\begin{tabular}{|l|l|l|l|}
\hline Año & País & Nombre & Observación \\
\hline 1980 & $\begin{array}{l}\text { Estados } \\
\text { Unidos }\end{array}$ & $\begin{array}{l}\text { Creación del Mando de Operaciones } \\
\text { Especiales Conjunto de Estados Unidos, } \\
\text { JSOC. }\end{array}$ & $\begin{array}{l}\text { Estructura } \\
\text { Conjunta }\end{array}$ \\
\hline 1981 & Noruega & $\begin{array}{l}\text { Mando especial de la Defensa noruega } \\
\text { (FSK) }\end{array}$ & $\begin{array}{l}\text { Estructura } \\
\text { Conjunta }\end{array}$ \\
\hline 1987 & $\begin{array}{l}\text { Estados } \\
\text { Unidos }\end{array}$ & $\begin{array}{l}\text { Mando de Operaciones especiales de de } \\
\text { EE.UU. (USSOCOM) }\end{array}$ & $\begin{array}{l}\text { Estructura } \\
\text { Conjunta }\end{array}$ \\
\hline 1987 & $\begin{array}{l}\text { Reino } \\
\text { Unido }\end{array}$ & $\begin{array}{l}\text { Dirección de fuerzas especiales del Reino } \\
\text { Unido (DSF) }\end{array}$ & $\begin{array}{l}\text { Estructura } \\
\text { Conjunta }\end{array}$ \\
\hline 1996 & Flemancia & $\begin{array}{l}\text { Mando de operaciones especiales de de } \\
\text { Francia. }\end{array}$ & $\begin{array}{l}\text { Estructura } \\
\text { Conjunta }\end{array}$ \\
\hline 1998 & $\begin{array}{l}\text { España } \\
\text { Mando de fuerzas especiales (KSK) }\end{array}$ & $\begin{array}{l}\text { Estructura } \\
\text { Ejercito }\end{array}$ \\
\hline
\end{tabular}

${ }^{27}$ Horowitz, Michael C. (2010): The Diffusion of Military Power: Causes and Consequences for International Politics, Princeton, Princeton University Press. 


\begin{tabular}{|l|l|l|l|}
\hline 2000 & Holanda & $\begin{array}{l}\text { Departamento de operaciones especiales de } \\
\text { Holanda. }\end{array}$ & $\begin{array}{l}\text { Estructura } \\
\text { Conjunta }\end{array}$ \\
\hline
\end{tabular}

Fuente: elaboración propia basado en información de NATO Special Operations Headquarters, Special Operations Forces Study. December 2012; USSOCOM Fact Book 2013; USSOCOM, United States Special Operations Command 20th Anniversary History, 2007.

Si en casi veinte años, de 1981 al 2000, habían visto la luz siete nuevas organizaciones de fuerzas de operaciones especiales, pertenecientes a siete países, del 2002 al 2014, en casi la mitad de tiempo, lo habían hecho 19 nuevas estructuras, pertenecientes a 12 países y a una organización internacional. Es decir, desde 2001, se había creado el doble de estructuras de operaciones especiales en la mitad de años. Una muestra de emulación que refleja la importancia y la utilidad concedida desde el ámbito internacional a las capacidades de operaciones especiales.

Cuadro 2. Creación de estructuras orgánicas y unidades por países occidentales a partir de 2001

\begin{tabular}{|c|c|c|c|}
\hline Año & País & Nombre & Observación \\
\hline 2002 & Brasil $\theta$ & $\begin{array}{l}\text { Creación de una Brigada de fuerzas } \\
\text { especiales }\end{array}$ & $\begin{array}{l}\text { Estructura } \\
\text { Ejercito }\end{array}$ \\
\hline 2002 & Francia & Brigada de operaciones especiales del ET & $\begin{array}{l}\text { Estructura } \\
\text { Ejército }\end{array}$ \\
\hline 2003 & Australia & $\begin{array}{l}\text { Mando de operaciones especiales de } \\
\text { Australia. (SOCOMD) }\end{array}$ & $\begin{array}{l}\text { Estructura } \\
\text { Conjunta }\end{array}$ \\
\hline 2004 & Italia & $\begin{array}{l}\text { Mando conjunto de operaciones especiales } \\
\text { de Italia (COFS). }\end{array}$ & $\begin{array}{l}\text { Estructura } \\
\text { Conjunta }\end{array}$ \\
\hline 2005 & Alemania & $\begin{array}{l}\text { Mando conjunto de operaciones especiales } \\
\text { de Alemania (commando FOSK) }\end{array}$ & $\begin{array}{l}\text { Estructura } \\
\text { Conjunta }\end{array}$ \\
\hline 2005 & EE:UU & $\begin{array}{l}\text { Centro para Operaciones Especiales, CSO } \\
\text { (organización en beneficio del JSOC) }\end{array}$ & $\begin{array}{l}\text { Estructura } \\
\text { Conjunta }\end{array}$ \\
\hline 2005 & España & $\begin{array}{l}\text { Mando de Guerra Naval Especial de la } \\
\text { Armada }\end{array}$ & $\begin{array}{l}\text { Estructura de } \\
\text { Ejército /Armada }\end{array}$ \\
\hline 2005 & $\begin{array}{l}\text { Reino } \\
\text { Unido }\end{array}$ & Regimiento de Reconocimiento Especial & Unidad de Ejercito \\
\hline 2006 & $\begin{array}{l}\text { Estados } \overline{\underline{\underline{\underline{\underline{\underline{\underline{E}}}}}}} \\
\text { Unidos }\end{array}$ & $\begin{array}{l}\text { Mando de operaciones especiales de los } \\
\text { Marines de Estados Unidos (MARSOC) }\end{array}$ & $\begin{array}{l}\text { Estructura } \\
\text { Ejercito }\end{array}$ \\
\hline 2006 & OTAN $-\frac{1}{2}-$ & $\begin{array}{l}\text { Centro Coordinador de Operaciones } \\
\text { Especiales de la OTAN }\end{array}$ & $\begin{array}{l}\text { Estructura } \\
\text { multinacional }\end{array}$ \\
\hline
\end{tabular}




\begin{tabular}{|c|c|c|c|}
\hline 2006 & Canadá $*$ & $\begin{array}{l}\text { Mando de operaciones especiales de } \\
\text { Canadá (CANSOFCOM) }\end{array}$ & $\begin{array}{l}\text { Estructura } \\
\text { Conjunta }\end{array}$ \\
\hline 2006 & ReinoUnidơ & Grupo de Apoyo a Fuerzas Especiales & $\begin{array}{l}\text { Estructura } \\
\text { Ejercito }\end{array}$ \\
\hline 2007 & España & $\begin{array}{l}\text { Departamento de operaciones especiales } \\
\text { (J3B) en el Mando de Operaciones } \\
\text { (MOPS) }\end{array}$ & $\begin{array}{l}\text { Estructura } \\
\text { Conjunta }\end{array}$ \\
\hline 2007 & Polonia & $\begin{array}{l}\text { Mando de operaciones especiales de } \\
\text { Polonia (POLSOCOM) }\end{array}$ & $\begin{array}{l}\text { Creación de un } 4^{\circ} \\
\text { Ejercito/Servicio }\end{array}$ \\
\hline 2008 & Alemania $=$ & $\begin{array}{l}\text { Creación de una División OE que engloba } \\
\text { al KSK }\end{array}$ & $\begin{array}{l}\text { Estructura } \\
\text { Ejercito }\end{array}$ \\
\hline 2008 & Lituania & $\begin{array}{l}\text { Mando De Operaciones Especiales de } \\
\text { Lituania (LITHSOCOM) }\end{array}$ & $\begin{array}{l}\text { Estructura } \\
\text { Conjunta }\end{array}$ \\
\hline 2010 & OTAN $-4-4$ & $\begin{array}{l}\text { NSHQ, Cuartel General de OE de la } \\
\text { OTAN }\end{array}$ & $\begin{array}{l}\text { Estructura conjunta } \\
\text { y multinacional }\end{array}$ \\
\hline 2011 & Israel & Mando Conjunto de OE. Depth Corps & $\begin{array}{l}\text { Estructura } \\
\text { Conjunta }\end{array}$ \\
\hline 2014 & España $=$ & $\begin{array}{l}\text { Mando Conjunto de Operaciones } \\
\text { Especiales }\end{array}$ & $\begin{array}{l}\text { Estructura } \\
\text { Conjunta }\end{array}$ \\
\hline 2014 & Italia I I & Mando de Operaciones Especiales del ET & $\begin{array}{l}\text { Estructura } \\
\text { Ejército }\end{array}$ \\
\hline
\end{tabular}

Fuente: elaboración propia con información proporcionada por fuentes abiertas del Centro Coordinación de Operaciones Especiales y de Cuartel General de Operaciones Especiales de la OTAN en SHAPE, Mons, Bélgica, completada con entrevistas con oficiales de enlace internacionales en el Ejército de Tierra de España.

\subsection{Cambios en el entorno estratégico}

Como ya hemos señalado, un conjunto de factores que impulsan tanto los procesos de innovación militar, como los de emulación y difusión militar, son los cambios en el entorno estratégico, que demandan de las fuerzas armadas una adaptación a las nuevas necesidades. Esto se aprecia claramente en el papel cada vez más relevante que se otorga a las fuerzas de operaciones especiales. De manera resumida dichos factores son los siguientes:

Por un lado, el carácter en ocasiones asimétrico y en otras ocasiones híbrido de la amenaza a la que se enfrentan las fuerzas armadas aliadas en escenarios de insurgencia como los que existen a día de hoy en Afganistán, en Oriente Medio y en el Sahel. Tanto para misiones de acción directa (neutralización de cuadros intermedios y líderes de alto nivel de organizaciones terroristas, o rescate de rehenes), como para acciones indirectas vinculadas a la reforma del sector de la seguridad en misiones de estabilización.

Por otro, las opiniones públicas occidentales son cada vez menos tolerantes a los despliegues prolongados de grandes contingentes militares en países donde no siempre son 
bien acogidos y donde los objetivos a alcanzar resultan elusivos. El apoyo social disminuye más aún si la misión incluye el combate y la pérdida de vidas humanas. La lucha contra la insurgencia en Irak, con más de cuatro mil muertos en el lado americano, y la de Afganistán con otros dos mil soldados estadounidenses caídos son recordatorios del coste en bajas que suponen este tipo de despliegues. ${ }^{28} \mathrm{Al}$ mismo tiempo, la crisis económica y los recortes que ésta ha supuesto en las fuerzas armadas de la mayoría de los países OTAN tampoco hacen sostenible los despliegues militares a gran escala.

Todo ello está motivando una actitud más favorable en algunos gobiernos aliados al empleo preferente de las fuerzas de operaciones especiales. Se percibe positivamente y se acepta con más facilidad el despliegue de fuerzas de operaciones especiales a la hora de fortalecer las capacidades de fuerzas de seguridad locales. A la vez, las misiones de asistencia de fuerzas de seguridad locales y las actividades relacionadas con la reforma del sector de seguridad reciben menos atención por parte de la opinión pública y, en caso de conocerse, mayor aprobación. Desde el nivel político se aceptan igualmente con interés las capacidades de operaciones especiales por ofrecer un perfil muy discreto de empleo de la fuerza, que alarma menos a las opiniones públicas nacionales e internacionales y a las poblaciones locales (al ejercer una presencia poco intrusiva). Su limitada entidad las hace también rentables económicamente, al implicar menores costes su despliegue, y no estar supeditadas a grandes esfuerzos logísticos de sostenimiento. Por último, la esfera de decisión política entiende que se trata de una herramienta diferente para problemas y soluciones diferentes, que se adapta mejor en la lucha contra adversarios híbridos y asimétricos. ${ }^{29}$

Se imponen, por tanto, de forma gradual unos procesos de empleo de la fuerza con tres características fundamentales: pequeñas huellas logísticas y bajo nivel de presencia; muy alta capacidad de respuesta; y capacidad de asistencia y asesoramiento de las fuerzas locales. Tres características propias de las fuerzas de operaciones especiales.

En la mayoría de los países occidentales de referencia se están llevando a cabo de forma acelerada unos procesos de transformación en las fuerzas armadas, con la tendencia común de una reducción cuantitativa de las fuerzas convencionales terrestres (La disminución en cantidad está siendo compensada e con un incremento de la calidad en su preparación, equipamiento y tecnología) y aumento en las capacidades conjuntas de operaciones especiales. Los gráficos 3 y 4, con un análisis comparativo entre nueve países de la OTAN muestran que en un periodo medido desde 1996 hasta 2020 las fuerzas terrestres se habrán reducido previsiblemente en un 25\%. Mientras que de 2001 a 2020 las fuerzas de operaciones especiales habrán crecido casi un $200 \%$.

\footnotetext{
${ }^{28}$ Datos extraídos de Icasualties.org: http://icasualties.org/.

${ }^{29}$ Robinson: "The Future of Special Operations. Beyond Kill and Capture", op. cit.
} 
Gráfico 3. Datos comparativos sobre reducciones de fuerzas terrestres y aumento de capacidades de operaciones especiales en países occidentales de referencia y en otros países con menor producto interior bruto que España ${ }^{30}$

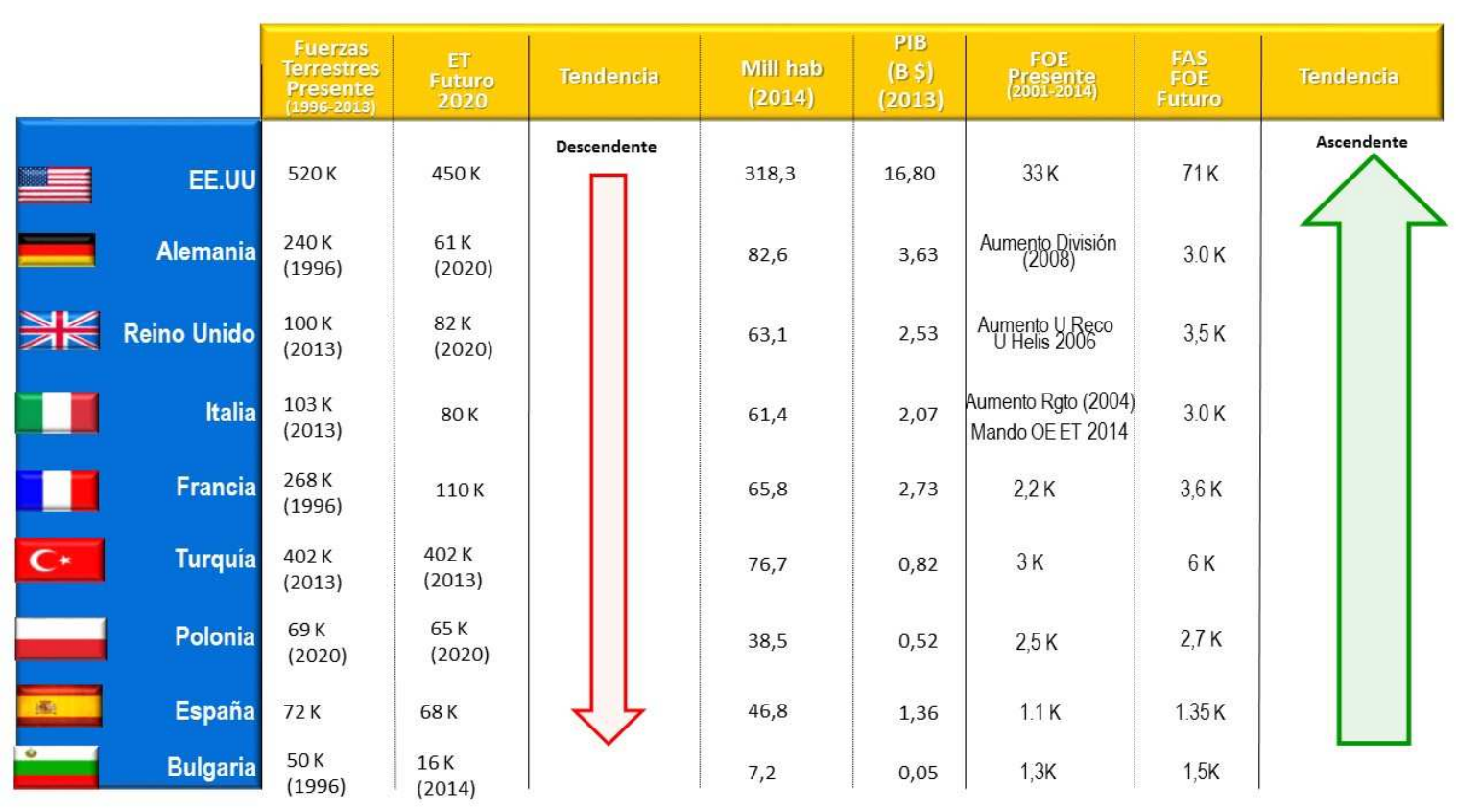

Fuente elaboración propia con Datos del Military Balance y de entrevistas con Oficiales de Enlace internacionales en el Ejército de Tierra de España; USSOCOM a través de página oficial. USSOCOM, Fiscal Year 2015 Budget Highlights; Feickert, Andrew, US Special Operations Forces (SOF): Background and issues for Congress, CRS Report for Congress, May 8, 2014.

Los esfuerzos futuros para mitigar el impacto de la reducción del tamaño de las fuerzas armadas en general, y de las fuerzas terrestres en particular, incluyen entre otras medidas un mayor protagonismo de las fuerzas de operaciones especiales. ${ }^{31}$ En concreto una elevada confianza en unas relativamente reducidas, pero cada vez más caras fuerzas de operaciones especiales con capacidad de llevar a cabo misiones muy específicas ${ }^{32}$.

\footnotetext{
${ }^{30}$ Los datos de España referidos a las Fuerzas de Operaciones Especiales (FOE) no incluyen fuerzas aeromóviles de ala rotatoria o fija.

${ }^{31}$ Lasconjarias, Guillaume: NATO's Land Forces: Losing Ground, Washington D.C. American Enterprise Institute for Public Policy Research (Junio de 2014).

${ }^{32}$ Esta tendencia queda reflejada con el establecimiento del Cuartel General de Operaciones Especiales de la OTAN (NATO Special Operations Headquarters, NSHQ). De acuerdo a su sitio web su establecimiento junto al Mando principal de Operaciones aliado (Allied Command Operations, ACO) confirma la función del NSHQ y su apoyo en asesoramiento sobre operaciones Especiales a SACEUR.
} 
Grafico 4. Tendencias de Incremento de Fuerzas de Operaciones Especiales en los últimos años.

Comparación de Volumen de Fuerzas

entre Fuerzas Terrestres y de Operaciones Especiales

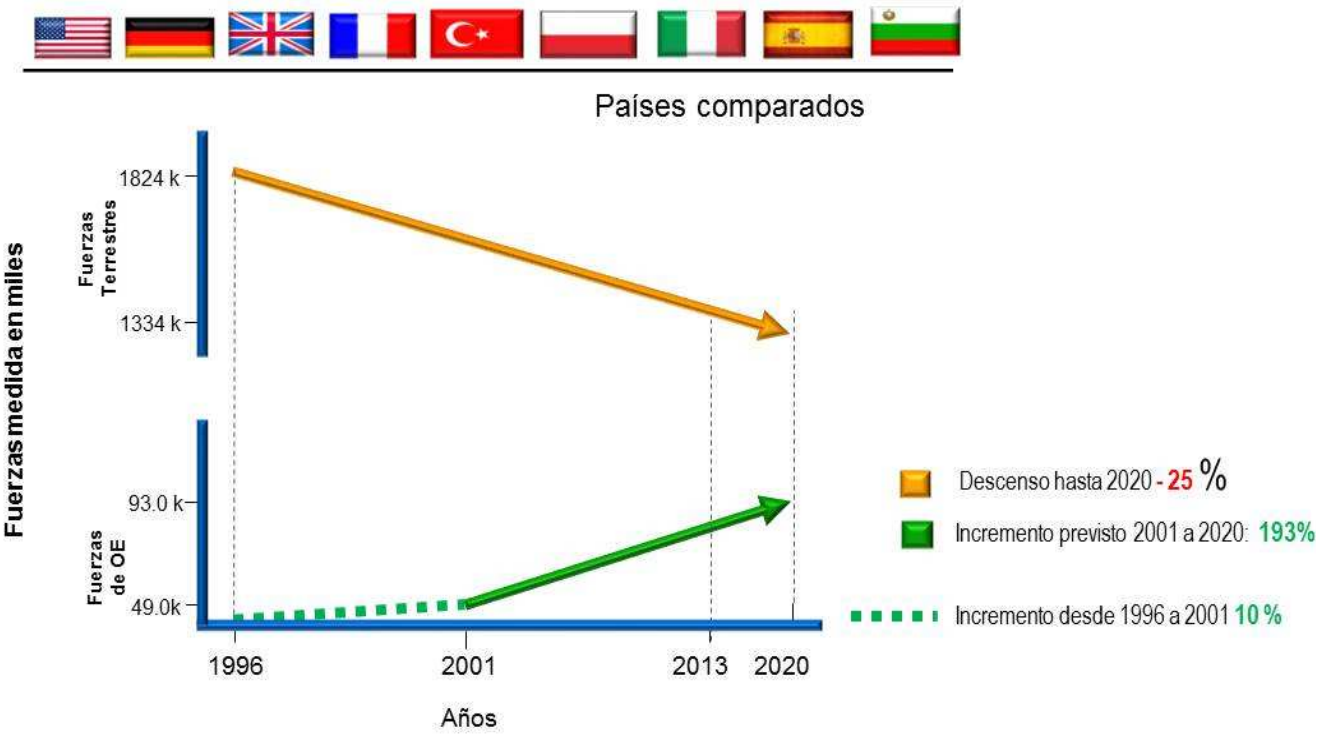

Fuente elaboración propia con Datos del Military Balance y de entrevistas con Oficiales de Enlace internacionales en el Ejército de Tierra de España; USSOCOM a través de página oficial. USSOCOM, Fiscal Year 2015 Budget Highlights; Feickert, Andrew, US Special Operations Forces (SOF): Background and issues for Congress, CRS Report for Congress, May 8, 2014.

Por otra parte, las fuerzas de operaciones especiales se adaptan con mayor facilidad a un entorno estratégico emergente cada vez más interconectado. Se trata de un modelo de empleo de fuerza más ágil y participativo, que favorece la colaboración e integración de capacidades. Entre las soluciones para optimizar el empleo de la fuerza, Estados Unidos ha desarrollado el concepto de «Global SOF Network». Esta iniciativa, ha experimentado un impulso muy importante durante los últimos años y a ella se están sumando fuerzas de todos los países del mundo. Para España supone una fuente de oportunidades de optimización de recursos y preparación de la fuerza muy importante.

La iniciativa Global SOF Network representa un nuevo modelo de cooperación internacional, en función de los intereses nacionales. Favorece la capacidad de respuesta rápida al establecer canales de relación más rápidos y eficaces que los tradicionales de cooperación internacional de alianzas, los cuales con mecanismos de relación más burocráticos, precisan de consenso, tienen ciclos de decisión más largos, corriendo el riesgo de ofrecer respuestas menos eficaces.

Para los países de limitada entidad económica y nivel de ambición político medio ha supuesto una buena inversión el despliegue de fuerzas de operaciones especiales en Afganistán. Por ejemplo, Lituania, Bélgica u Holanda han logrado una mayor visibilidad y unos buenos retornos en términos de influencia internacional con el empleo de pequeños compromisos de fuerza de operaciones especiales ${ }^{33}$.

\footnotetext{
${ }^{33}$ Holanda participa en la actualidad con un contingente de más de 300 militares de fuerzas especiales, en Mali, bajo mando de MINUSMA. La postura holandesa de compromiso consigue mucha visibilidad a los ojos de la
} 
En realidad, la rentabilidad del binomio eficacia-coste económico de fuerzas de operaciones especiales es siempre alto. Son unidades muy baratas comparadas con otras. El coste de un Eurofighter es de aproximadamente 77 millones de euros, el de un helicóptero NH-90 16 millones y el de un avión de transporte A-400M 100 millones de euros, mientras que el precio de una fragata europea multi- misión (FREMM) es de aproximadamente unos 342 millones. Comparativamente, esas cantidades quedan muy por encima de la inversión de 13 millones de euros que requiere equipar completamente a unos 110 militares de operaciones especiales, incluyendo la movilidad a base de vehículos especiales, comunicaciones, ordenadores, armamento, visión nocturna, vigilancia óptica y otro tipo de equipo especial. Es decir, un Grupo de Operaciones Especiales de unos 250 efectivos con tecnología de última generación, con el apoyo necesario y mantenimiento para personal, equipo y armas costaría aproximadamente 26 millones de euros.

En relación a los presupuestos de 2007 de diferentes países OTAN esa cantidad de 26 millones de euros supone un 5\% del presupuesto militar estonio, 3\% del Lituania o Bélgica, el $2 \%$ del de Eslovenia, el 1\% del de Eslovaquia, Polonia y Hungría y muy por debajo del 0,01 $\%$ de los gastos de Defensa de países como Alemania, Italia, Francia, Canadá, España, Reino Unido o los Estados Unidos. ${ }^{34}$ Estudios y valoraciones que no escapan del seguimiento de las comisiones de defensa de los parlamentos de los países OTAN, quienes cada vez más ejercen un mayor control en los gastos de defensa y en el análisis de su rentabilidad futura.

\subsection{La operación en Afganistán como impulsor de la difusión militar}

Otro factor que ha contribuido a la importancia creciente de las fuerzas de operaciones especiales de los países aliados ha sido su formidable empleo en Afganistán, en el marco de la ISAF. Inicialmente la presencia se limitó a países como Polonia, Reino Unido, Francia y Dinamarca y otros aliados no OTAN como Australia o algunos países árabes que comprometieron sus fuerzas en la operación Enduring Freedom. ${ }^{35} \mathrm{El}$ incremento de este tipo fuerzas sucedería a partir de 2006, tras la creación de una estructura permanente de operaciones especiales en el ámbito de la Alianza. ${ }^{36}$

Ese mismo año la OTAN había reconocido expresamente el mayor protagonismo atribuido a las fuerzas de operaciones especiales en un documento publicado por el Comité Militar. En dicho documento, el de mayor nivel que establece la política de operaciones especiales de la OTAN, se afirmaba que las fuerzas de operaciones especiales eran las más apropiadas para enfrentarse a las amenazas difusas a las que tenía que dar respuesta la OTAN, entre ellas las múltiples formas de terrorismo. ${ }^{37}$

En la cumbre de Riga de noviembre de 2006, los Jefes de Estado de los países miembros de la OTAN aprobaron el desarrollo de la denominada Iniciativa de

ONU. Su apuesta por un pequeño contingente de fuerzas, pero de elevada calidad, es una medida de gran rentabilidad para su aspiración de ocupar un espacio como miembro no permanente del Consejo de Seguridad de Naciones Unidas para el periodo 2017-2018.

${ }^{34}$ NATO Special Operations Headquarters: "Special Operations Forces Study" (Diciembre de 2012), p. 30.

${ }^{35}$ Carta del Ministro de Defensa de Polonia, Jerzy Szmajdzinski al Secretario de Defensa de Estados Unidos, Donald Rumsfeld, el 3 de Agosto de 2005, y contestaciones de Secretario de Defensa, así como Action Memo de Peter Floty, Vicesecretario de Defensa para Política de Seguridad Internacional, en http://papers.rumsfeld.com/library/.

${ }^{36}$ Chamberlain, Nigel: "New Special Forces HQ open for business", NATO Watch, 14 de diciembre de 2012, en http://www.natowatch.org/node/834.

${ }^{37}$ Las SOF están jugando un papel de creciente importancia en los escenarios asimétricos y son fuerzas muy apropiadas para contribuir a dar respuesta a las amenazas difusas a las que se enfrenta la OTAN, tales como las múltiples modalidades de terrorismo. NATO: "MC 437/1 Military Committee Special Operations Policy" (14 de junio de 2006), p. 3, citado en NATO Special Operations Headquarters, "Special Operations Forces Study" (Diciembre 2012), p. 10. 
Transformación de las Fuerzas de Operaciones Especiales de la OTAN (NATO Special Operations Transformation Initiative, NSTI). La buena marcha de esa iniciativa fue presentada por el Mando Supremo de la Alianza en Europa (SACEUR) como ejemplo de objetivos alcanzados en tiempo récord en la reunión de altos dignatarios de la OTAN celebrada en Bucarest en abril de 2008. Desde entonces la NSTI continuó evolucionando, en gran medida debido a la necesidad y el impulso que la OTAN proporcionó a las capacidades de operaciones especiales sobre el terreno, en Afganistán.

En 2006 el Almirante McRaven, Jefe del Mando de Operaciones Especiales en Europa, fue designado primer Jefe del Centro Coordinador de Operaciones Especiales en el ámbito de la OTAN (NSCC en sus iniciales inglesas). Su liderazgo en el desarrollo de esa iniciativa fue decisivo para el impulso que experimentó durante los primeros años. El NSCC constituía el embrión de lo que en 2010 llegaría ser un Cuartel General de Operaciones Especiales en la OTAN (NSHQ). McRaven tenía la intención de crear un lugar de enseñanza y adiestramiento común para los miembros de las fuerzas de operaciones especiales de la OTAN. Aprovechó la puesta en marcha del NSCC para fomentar la cultura de operaciones especiales e impulsar la participación de fuerzas especiales de los países Europeos en la Operación ISAF en Afganistán. Por ese motivo viajó por todos los países miembros tratando de conseguir un compromiso de implicación de fuerzas. ${ }^{38}$

La iniciativa de McRaven tuvo éxito y del año 2008 al 2012 el incremento de la presencia de fuerzas de operaciones especiales de la coalición multinacional de la operación ISAF en Afganistán ascendió en un $600 \%$. ${ }^{39}$ Llegaron a desplegar anualmente una media de veintiséis países, aportando en total unos 2.250 operadores. El número de militares desplegados y de países variaba cada año. Las fuerzas de operaciones especiales son un recurso limitado y ello dificulta que todos los países puedan mantener una presencia continuada con rotaciones sostenidas. Algunos, como Noruega, Suecia, y Lituania, alternaban periodos de presencia con periodos de descanso. Las cifras oscilaban ligeramente de un año a otro. El gráfico 5 muestra el número de países y de fuerzas desplegadas en el periodo comprendido entre el año 2011 al 2012. Los Estados Unidos mantenían en la operación ISAF una presencia testimonial de 150 operadores. El grueso de sus fuerzas se encontraba bajo mandato de la operación Enduring Freedom, con reglas de enfrentamiento menos restrictivas que ISAF, lo que les proporcionaba mayor flexibilidad.

\footnotetext{
${ }^{38}$ Robinson, "One hundred victories...", op. cit.

39 "NATO Special Operations Headquarters", op. cit, p. i.
} 
Grafico 5. Número de países y fuerzas de operaciones especiales desplegadas en la operación ISAF entre los años 2011 y 2012

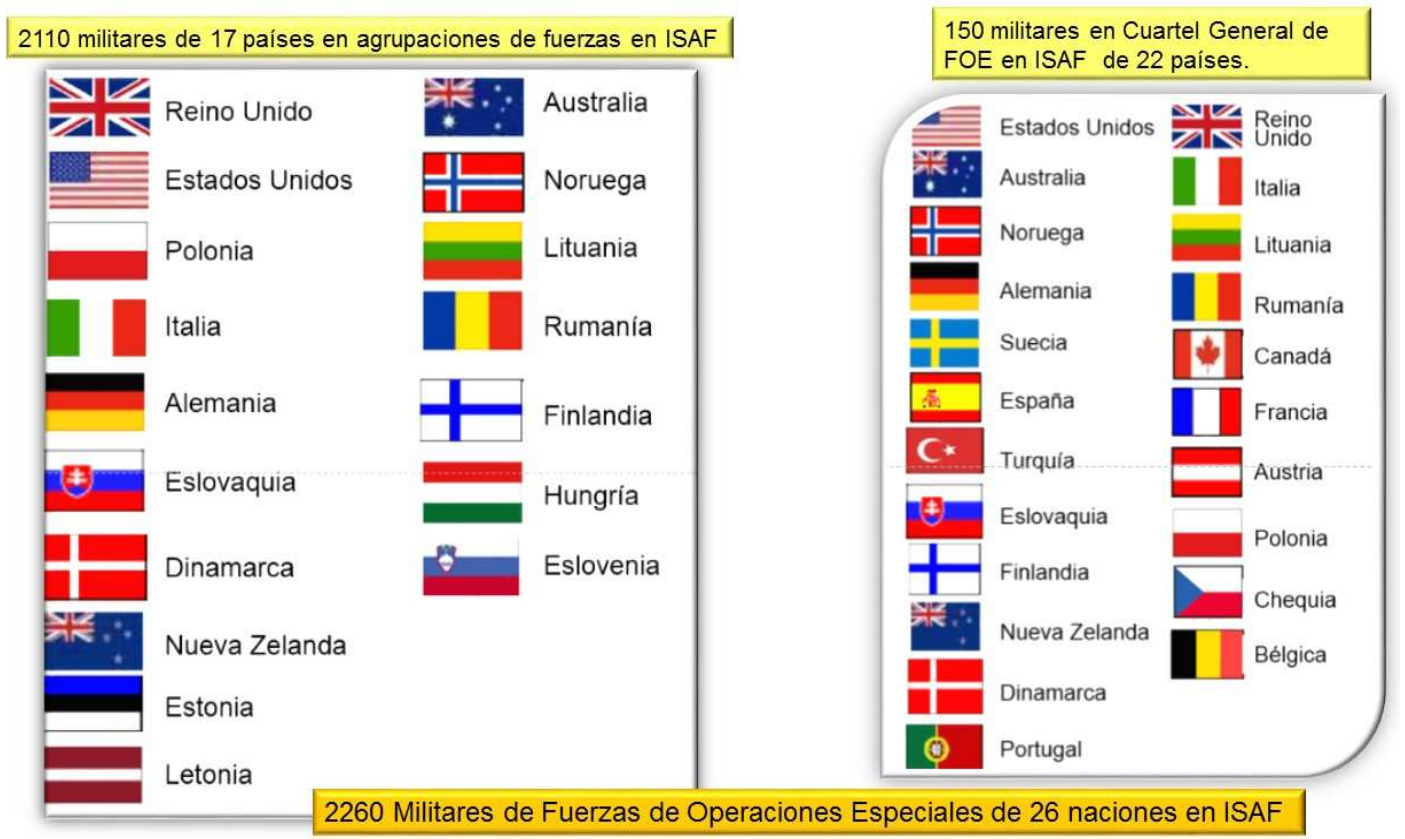

Fuente: elaboración propia basado en datos de: Morgan, Wesley: Coalition Combat Forces in Afghanistán. Afghanistan order of Battle, Institute for the Study of War, April 2013, en

https://www.understandingwar.org/sites/default/files/AfghanistanOrbat_April2013.pdf; Brigadier General Mark Smethurst (AUS) COM ISAF SOF: «Brief to SO/LIC Symposium» 11-12 de octubre de 2013. Disponible en http://www.dtic.mil/ndia/2013SOLIC/Smethurst.pdf.

Las fuerzas de operaciones especiales encuadradas en la operación de ISAF operaron en Afganistán hasta 2012 bajo un solo mando, dependientes de un Cuartel General denominado NATO SOF HQ, cuyo Jefe era un General de Brigada, normalmente americano, británico o australiano. El Jefe del NATO SOF HQ informaba y dependía directamente del Comandante del Teatro (COMISAF), aspecto que simplificaba sustancialmente las relaciones de mando. Un mando único de operaciones especiales en la cadena de ISAF que favorecía la sincronización y el aprovechamiento de los medios disponibles en teatro.

Por tanto, en Afganistán existían dos cadenas de Mando de fuerzas de operaciones especiales: la de Enduring Freedom y la de ISAF, entre las que había cada vez un mayor grado de entendimiento y coordinación; lo que a su vez favoreció una buena unidad de esfuerzo. Entre ambas cadenas de mando se compartían medios y productos de inteligencia, medios de vigilancia y reconocimiento (ISR), los helicópteros a disposición de la operación ISAF y los de la operación Enduring Freedom. Todas estas capacidades se integraron con las de las unidades de operaciones especiales afganas de reciente creación. Muchas de ellas habían sido las primeras de las fuerzas armadas afganas en alcanzar la capacidad de operar autónomamente sin el liderazgo de ningún país. La coordinación fue viable respetando los intereses nacionales de cada uno de los Estados y el mandato OTAN. A partir de 2012, se lograría la unidad de mando creándose un mando único detentado por un General americano de dos estrellas. ${ }^{40}$ Dicho mando terminó unificando definitivamente las cadenas de ISAF y Enduring Freedom.

\footnotetext{
${ }^{40}$ United States Special Operations Command, "Factbook 2013", en http://www.socom.mil/News/Documents/USSOCOM_Fact_Book_2013.pdf.
} 
Gráfico 6. Organización operativa de las fuerzas de Operaciones Especiales en Afganistán a partir de 2012, con cadena de mando única

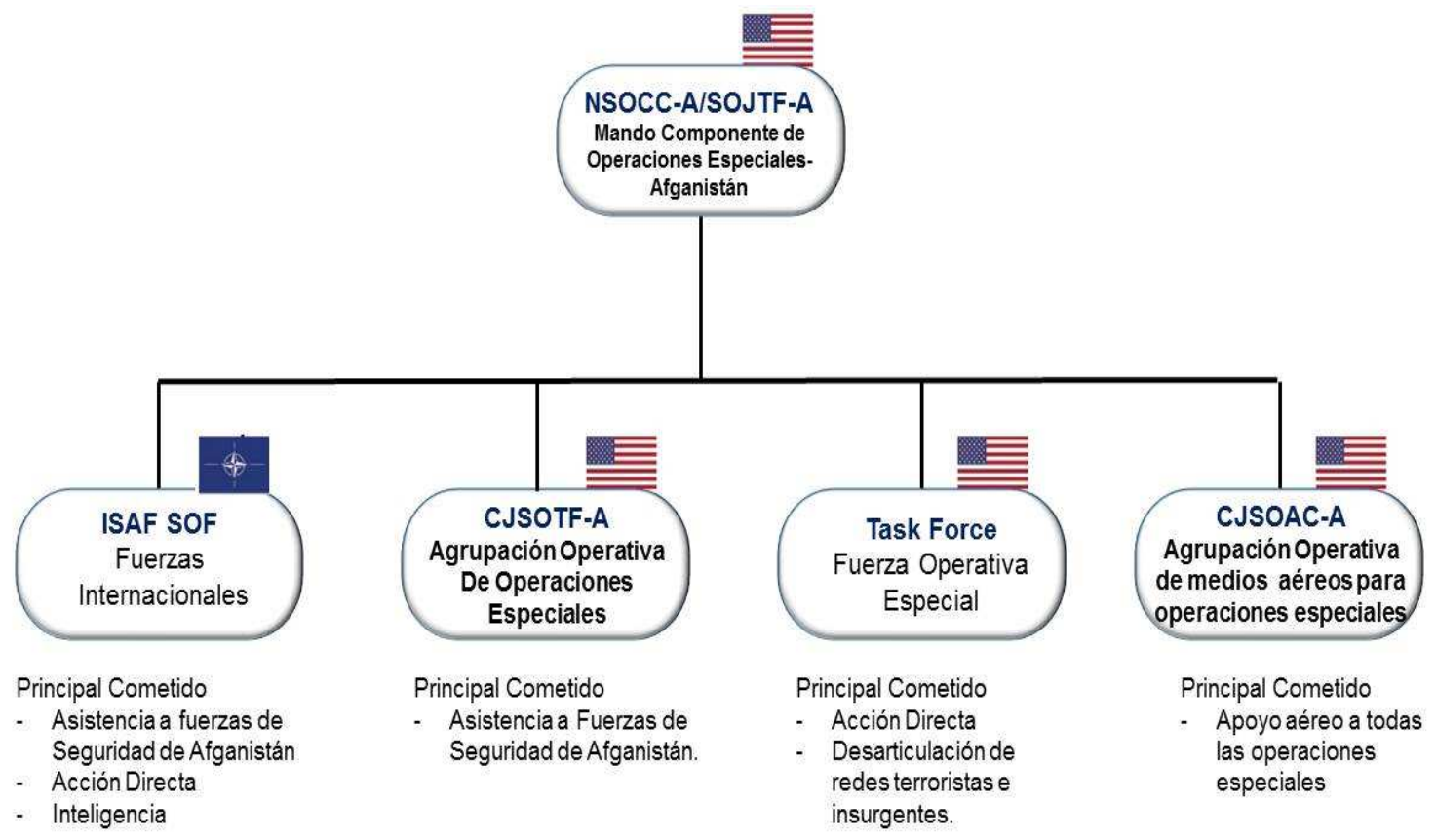

Fuente: Elaboración propia a partir de los Factbook 2013 y 2014 del United States Special Operations Command.

\section{Conclusión}

Los ejércitos son organizaciones vivas que responden a los inputs procedentes del entorno. En el caso que hemos analizado, tanto la naturaleza de la amenaza, como los condicionantes de carácter social, político y económico tienden a potenciar el protagonismo de las unidades de operaciones especiales en las fuerzas armadas de los países OTAN. Unas fuerzas que tradicionalmente han ocupado un rol secundario y en apoyo del grueso de las fuerzas convencionales, pero que desde hace algo más de una década han visto elevado su perfil, también en términos de recursos humanos y materiales. La continuidad de los factores que han impulsado este proceso de innovación militar apunta a la pervivencia y profundización de este proceso de cambio. 